\title{
An unusual Sign of Superior Vena Cava Obstruction: CT Quadrate Lobe Hot Spot Sign
}

\author{
Karthik Marpalli Vasudeva ${ }^{1}$, Hamsa $\mathrm{K}^{2}$ \\ ${ }^{1}$ Assistant professor, Department of Radiodiagnosis, A.J. Institute of Medical Sciences and Research Centre, Mangalore,

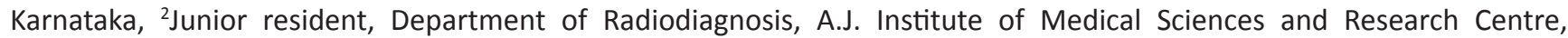 \\ Mangalore, Karnataka, India
}

Corresponding author: Karthik Marpalli Vasudeva, Assistant professor, Department of Radiodiagnosis, A.J. Institute of Medical Sciences and Research Centre, Mangalore, Karnataka, India

DOI: http://dx.doi.org/10.21276/ijcmsr.2020.5.2.19

How to cite this article: Karthik Marpalli Vasudeva, Hamsa K. An unusual sign of superior vena cava obstruction: CT quadrate lobe hot spot sign. International Journal of Contemporary Medicine Surgery and Radiology. 2020;5(2):B79-B80.

\section{A B S T R A C T}

Introduction: A contrast-enhanced Computed Tomography scan of the abdomen can show enhancement of the Quadrate lobe of the liver in cases of superior vena caval obstruction similar to the technetium ${ }^{99 m}$ sulfur colloid scan.

Case report: A 68-year-old male with complaints of generalized weakness, loss of appetite, weight loss and diffuse dull aching abdominal pain in the last 1 month, and 1-week history of swollen face and marked venous distension over the chest presented to the emergency department. Contrast-enhanced computed tomography of the chest and the whole abdomen performed revealed an enhancing lobulated mass lesion in the apex of the right lung with compression of proximal superior vena cava and the confluence of right / left brachiocephalic veins with an irregular area of intense arterial phase enhancement in the quadrate lobe that represented the CT "Quadrate lobe hot spot" sign.

Conclusion: The presence of a CT quadrate lobe hot spot sign in the liver with multiple collaterals can be a sign of lung tumors causing SVC obstruction.

Keywords: CT Quadrate Lobe Hot Spot Sign, SVC obstruction

\section{INTRODUCTION}

A contrast-enhanced Computed Tomography scan of the abdomen can show enhancement of the Quadrate lobe of the liver in cases of superior vena caval obstruction similar to the technetium ${ }^{99 \mathrm{~m}}$ sulfur colloid (TSC) scan. This sign is believed to be caused by the portosystemic venous shunting between the superior vena cava and the portal vein within the liver. We present such a sign, in case of lung carcinoma with SVC obstruction and importance of this sign.

\section{CASE REPORT}

A 68-year-old male with complaints of generalized weakness, loss of appetite, weight loss and diffuse dull aching abdominal pain in the last 1 month, and 1-week history of swollen face and marked venous distension over the chest presented to the emergency department. On clinical examination, the patient had tachycardia and tachypnoea. Chest radiography showed opacity in the apex of the right lung. Contrast-enhanced computed tomography (CECT) of the chest and whole abdomen was performed. Imaging revealed an enhancing lobulated mass lesion in the apex of the right lung (Figure 1) with compression of proximal superior vena cava and confluence of right / left brachiocephalic veins (Figure 2). Multiple anterior chest wall and abdominal wall collaterals were also seen. An irregular area of intense arterial phase enhancement (CT Quadrate lobe hot spot sign) in segment IV of the liver was noted (Figure 3). This area of enhancement showed complete washout in the delayed phase imaging. The patient underwent a percutaneous biopsy of the lung lesion and the histopathology report suggested the diagnosis of squamous cell carcinoma.

\section{DISCUSSION}

The CT Quadrate lobe Hot Spot sign was first defined in $1983 .{ }^{1}$ It is usually described as a wedge-shaped area of intense enhancement of the quadrate lobe of the liver. The characteristic location helps differentiate from other hypervascular liver lesions. It is caused due to portosystemic shunting between the SVC and the left portal vein. ${ }^{2}$

Focal hot spot sign can also be caused by hepatic hemangiomas, abscesses, focal nodular hyperplasia, and hepatocellular carcinomas in any part of the liver. ${ }^{3}$

In our case, the characteristic site of enhancement in the liver helped us to diagnose it as secondary to SVC obstruction rather than metastasis or hepatic hemangiomas. This sign usually occurs from direct tumor invasion of SVC, extrinsic compression, or thrombosis secondary to the slowing and stagnating of blood as a result of a partial obstruction. ${ }^{4}$ In cases of clinically unapparent obstruction of SVC, this sign assumes importance because it provides clues to the diagnosis. ${ }^{2,5}$ 


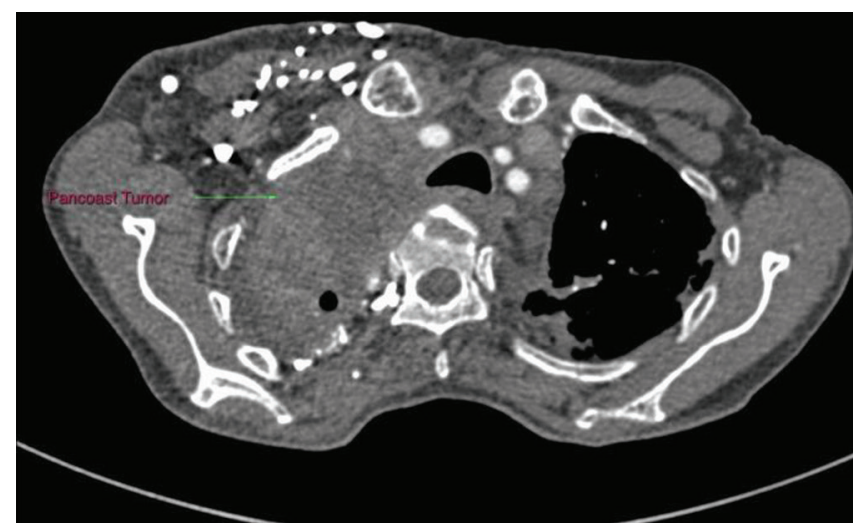

Figure-1: Contrast- enhanced CT scan of the thorax showing the Pancoast tumour in the right upper lobe.

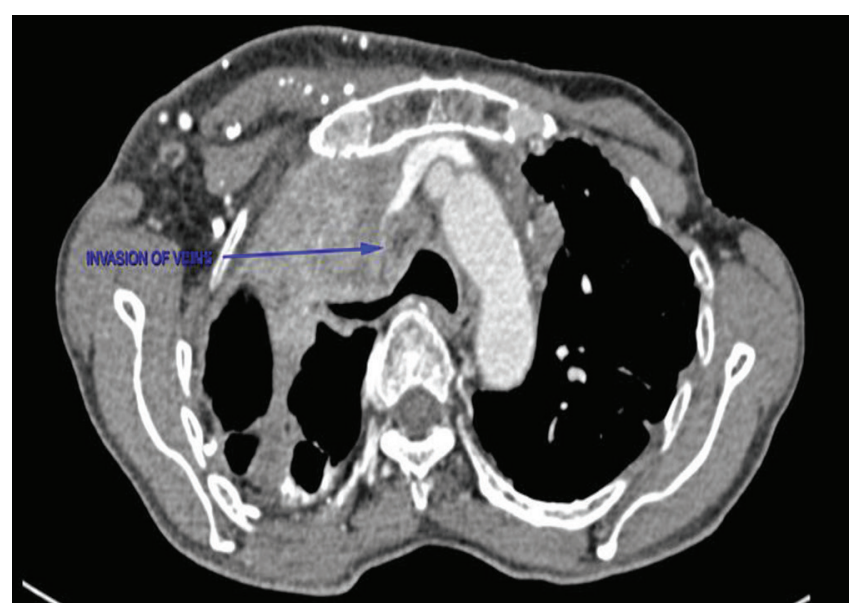

Figure-2: Contrast-Enhanced CT Scan of the Thorax Showing the Pancoast Tumour Invading the Superior Vena Cava.

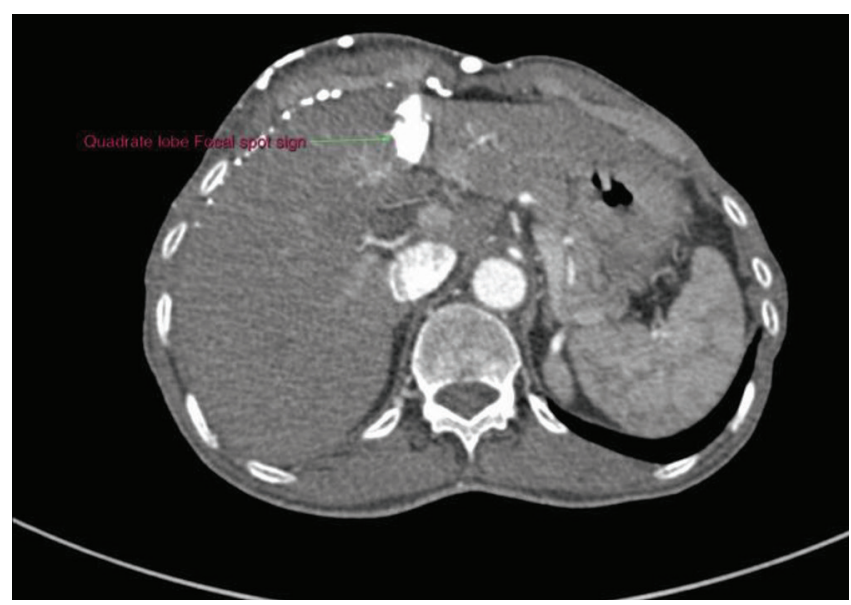

Figure-3: Contrast-Enhanced CT Scan Of the Thorax showing intense enhancement of the quadrate lobe (arrow) in the early arterial phase suggestive of CT Quadrate lobe hot spot sign.

\section{CONCLUSION}

A contrast-enhanced CT scan of the abdomen can show enhancement of the Quadrate lobe of the liver in a variety of causes. The presence of a quadrate lobe enhancement with multiple collaterals can be a sign of lung tumors causing SVC obstruction.

\section{REFERENCES}

1. Sureka B, Sullere A, Singh Khera P. CT Quadrate Lobe Hot Spot Sign. Middle East J Dig Dis. 2018;10(3):192193.

2. Dickson, A.M. The focal hepatic hot spot sign. Radiology 2005;237(2):647-648.

3. Muramatsu T, Miyamae T, Mashimo M, Suzuki K, Kinoshita S, Dohi Y. Hot spots on liver scans associated with superior or inferior vena caval obstruction. Clin Nucl Med 1994;19(3):622- 629

4. Stanford, W., Jolles, H., Ell, S. and Chiu, L.C. Superior vena cava obstruction: a venographic classification. American Journal of Roentgenology, 1987;148(2), pp.259-262.

5. Virmani, Vivek, Anupam Lai, Chirag K. Ahuja, and Niranjan Khandelwal.The CT Quadrate Lobe Hot Spot Sign. Annals of Hepatology 2010;9(5):296-98.

\section{Source of Support: Nil; Conflict of Interest: None}

Submitted: 19-04-2020; Accepted: 29-05-2020; Published online: 26-06-2020 